Revue

Revue de l'histoire des religions

de Ihistoire des religions

\title{
Critique textuelle et expérience mystique : la série thérésienne de Claude Langlois
}

Notes critiques

Textual criticism and mystical experience : Claude Langlois' Theresian series

Anthony Feneuil

\section{(2) OpenEdition}

Journals

Édition électronique

URL : http://journals.openedition.org/rhr/7717

DOI : $10.4000 /$ rhr.7717

ISSN : 2105-2573

Éditeur

Armand Colin

Édition imprimée

Date de publication : 1 mars 2011

Pagination : 93-103

ISBN : 978-2200-92685-4

ISSN : 0035-1423

Référence électronique

Anthony Feneuil, «Critique textuelle et expérience mystique : la série thérésienne de Claude Langlois », Revue de I'histoire des religions [En ligne], 1 | 2011, mis en ligne le 01 mars 2014, consulté le 11 octobre 2020. URL : http://journals.openedition.org/rhr/7717 ; DOI : https://doi.org/10.4000/rhr.7717 
ANTHONY FENEUIL

Université de Genève

Faculté de théologie - IRSE

\section{Critique textuelle et expérience mystique : la série thérésienne de Claude Langlois Notes critiques*}

Les six livres de Claude Langlois consacrés à Thérèse de Lisieux renouvellent de façon décisive les études sur le sujet. L'attention à la matérialité des textes de la sainte, l'application systématique qui leur est faite des méthodes de l'histoire critique et l'édition critique des plus importants d'entre eux donnent à voir une nouvelle Thérèse de Lisieux. En révélant une Thérèse écrivain, la critique textuelle ne reste pas extérieure à son expérience spirituelle, mais en dévoile au contraire une part essentielle, celle qui se joue pour elle dans l'écriture.

Textual criticism and mystical experience : Claude Langlois' Theresian series

The six books that Claude Langlois devoted to Therese of Lisieux constitute a decisive renewal for studies on the topic. The focus on the material side of the saint's texts, the systematic application of historical criticism to them, and the critical edition of the most important of these texts show a new Therese of Lisieux. By revealing Therese as a writer, textual criticism does not disregard her spiritual experience, but unveils an essential part of it, the one which occurs in writing.

* À propos de: Claude Langlors: Les Dernières Paroles de Thérèse de Lisieux (Paris, Salvador, 2000, 173 p.); Le Désir de sacerdoce chez Thérèse de Lisieux (Paris, Salvador, 2002, 231 p.); Le Poème de septembre. Lecture du Manuscrit $B$ de Thérèse de Lisieux (Paris, Cerf, 2002, 241 p.); Lettres à ma Mère bienaimée. Juin 1897. Lecture du Manuscrit C de Thérèse de Lisieux (Paris, Cerf, 2007, 416 p.); L'Autobiographie de Thérèse de Lisieux. Édition critique du Manuscrit A (1895) (Paris, Cerf, 2009, 591 p.); Thérèse et Marie-Madeleine. La rivalité amoureuse (Paris, Jérôme Millon, 2009, 238 p.) 
En dix ans ont paru six livres de Claude Langlois sur Thérèse de Lisieux (1873-1897), fruits d'un travail entamé dès 1995 à l'occasion de séminaires à l'École pratique des hautes études. Près de deux mille pages visiblement écrites dans un même élan, mais qu'il faut regrouper en deux grands ensembles. Un diptyque, d'abord: Les Dernières Paroles de Thérèse de Lisieux (2000) et Le Désir de sacerdoce chez Thérèse de Lisieux (2002). Puis un triptyque : Le Poème de septembre. Lecture du Manuscrit B de Thérèse de Lisieux (2002), Lettres à ma Mère bien-aimée. Juin 1897. Lecture du Manuscrit C de Thérèse de Lisieux (2007), L'Autobiographie de Thérèse de Lisieux. Édition critique du Manuscrit A (1895) - ce dernier volume paru en 2009, en même temps qu'une nouvelle étude critique : Thérèse et Marie-Madeleine. La rivalité amoureuse. Toutefois, avec le diptyque initial s'annonce déjà toute l'entreprise de C. Langlois. De l'aveu de l'auteur, en effet, c'est la rédaction du Désir de sacerdoce chez Thérèse de Lisieux qui suscite la nécessité de l'étude sur ses dernières paroles : pour comprendre exactement la nature de son désir, il faut en effet s'entendre sur le crédit à accorder aux propos de Thérèse rapportés dans divers carnets par ses sœurs au Carmel et regroupés dans l'édition des CEuvres complètes sous le titre «Derniers entretiens ». Or très vite, cette étude dépasse son ambition strictement méthodologique pour donner un aperçu des derniers mois de la sœur et révéler « une Thérèse familière et singulière, qui cherchait à apprivoiser sa mort et à ruser avec la vie, à dire avec obstination ses angoisses, ses incertitudes, ses désirs ${ }^{2} »$.

Tel est bien l'apport le plus frappant de l'œuvre thérésien de C. Langlois : la démonstration de ce que la compréhension de la personnalité de Thérèse et de son expérience spirituelle passe par une étude critique de ses textes, non pas seulement au sens où la critique serait un préambule méthodologique indispensable pour solidement établir les informations qu'ils donnent, mais au sens où c'est tout un que comprendre Thérèse et saisir sa manière d'écrire. C'est pourquoi l'entreprise culmine dans les trois livres consacrés à ce que les premiers éditeurs scientifiques de l'œuvre de Thérèse ont appelé ses

2. Les Dernières paroles de Thérèse de Lisieux, éd. cit., p. 18. 
trois « Manuscrits autobiographiques », puisque si ce sont à chaque fois trois commentaires de textes, trois études approfondies sur la vie et la spiritualité de Thérèse de Lisieux, ce sont aussi trois nouvelles éditions des textes de la sainte.

Déjà dans l'ouvrage sur le Désir de sacerdoce, que l'on peut considérer comme le point de départ de tout le projet ${ }^{3}$, l'écriture thérésienne joue un rôle considérable. En étudiant le désir de sacerdoce, il s'agissait alors d'approcher la sainte par un aspect qui d'une part l'inscrit dans une longue série de spirituelles catholiques du $\mathrm{XIX}^{\mathrm{e}}$ siècle, et d'autre part déploie des enjeux actuels de politique ecclésiale qui nourrissent l'intérêt de C. Langlois, mais qu'il sait aussi mettre à distance lorsqu'ils portent le risque de lectures anachroniques des textes thérésiens ${ }^{4}$. Néanmoins, c'est bien dans l'écriture thérésienne que se joue l'essentiel : l'apaisement final des tensions constitutives du désir de sacerdoce, le passage d'un anticléricalisme vindicatif à la possibilité d'une fraternité avec les prêtres. Dans l'écriture, en effet, par le nouvel usage que Thérèse fait d'une image qu'elle connaît depuis longtemps : celle de Moïse obtenant par la prière la victoire de Josué contre les Amalécites, est révélée la manière dont elle assume finalement l'impossibilité d'être prêtre et sa position de retrait et de surplomb vis-à-vis d'eux. On pourrait peut-être dire, en allant un peu plus loin que C. Langlois dans ce livre, que cet usage n'est pas seulement le révélateur de l'apaisement du rapport de Thérèse aux prêtres, mais qu'il en est même la condition de possibilité, le biais par lequel Thérèse peut légitimer sa position et accepter la fraternité avec eux.

Reste que le livre sur les Dernières paroles est plus représentatif de la manière propre à Claude Langlois dans cette série. L'interrogation est portée sur le statut des textes - ici, divers carnets dans

3. Bien que publié deux ans après les Dernières paroles et la même année que Le Poème de septembre, dont il constitue d'ailleurs pour C. Langlois un prolongement (Le Désir de Sacerdoce..., op. cit., p. 16), il est issu de la première recherche menée par C. Langlois sur la sainte de Lisieux («Le statut incertain d'un véritable désir : Thérèse de Lisieux et le sacerdoce » dans Françoise Lautman (éd.), Ni Ève ni Marie. Luttes et incertitudes des héritières de la Bible, Genève, Labor et Fides, 1997, p. 101-119). À mon sens, il se situe par son contenu en amont des travaux sur les trois manuscrits bien que, par le thème du désir, il se rapporte directement à l'un d'entre eux et fasse évidemment usage des découvertes qui y sont faites.

4. C'est le cas, par exemple, de la problématique du sacerdoce des fidèles, introduite à Vatican II et qui reste étrangère à Thérèse. 
lesquels sont notées des " paroles 》 de Thérèse - reconstitué à partir de leur mode de production. Et l'élucidation progressive de ce mode de production ne va pas sans une compréhension fine des relations subtiles entre Thérèse et ses sœurs biologiques au Carmel : Marie, l'aînée, Céline et Pauline, auxquelles il faudrait ajouter la cousine de Thérèse, Marie. Les « dernières paroles » cessent alors d'apparaître comme le recueil transparent des paroles édifiantes d'une jeune carmélite qui « fait une belle mort». Elles prennent du relief en devenant plutôt le champ d'affrontement des motivations conscientes ou inconscientes des différents acteurs. Thérèse au centre, qui résiste comme elle peut à ses épreuves intérieures (doutes sur sa foi) et extérieures (tuberculose et questionnements aussi incessants que mortifères de sa sœur Pauline), au milieu de ses sœurs pour qui le recueil, parfois la sollicitation et, plus tard, la diffusion de ces paroles représentent un enjeu affectif majeur, dans les rapports qu'elles entretiennent avec leur cadette et entre elles. Les dernières paroles, finalement, comme enjeu et comme arme sans doute dans la partie complexe qui se joue au Carmel de Lisieux au moment de la mort de Thérèse - et après.

C'est de cette manière que sont envisagés les textes de Thérèse dans les trois ouvrages principaux de Claude Langlois : non pas comme des pièces informatives sur l'histoire d'une âme, mais comme un des lieux les plus décisifs pour l'accomplissement de cette histoire. De là l'importance de leur étude historico-critique. Pas pour vérifier la teneur des informations qu'ils transmettent, moins encore pour parvenir à un illusoire état de transparence primitive. Au contraire, en restituant les contextes d'écriture de ces textes, les étapes de leurs rédactions et les différents statuts que Thérèse leur confère, Claude Langlois leur rend une épaisseur, celle de la complexité de leurs rôles dans le développement social - aussi petite que soit la société de Thérèse -, affectif et spirituel de la moniale.

Ces trois ouvrages sont donc consacrés aux « Manuscrits autobiographiques ». Et justement, rendre à ces textes leur épaisseur en tâchant d'en comprendre le statut exact, c'est d'abord, pour C. Langlois, rompre avec cette appellation qui met sur un même plan trois manuscrits écrits à trois moments bien distincts dans une vie aussi courte $(1895,1896$ et 1897), adressés à trois personnes différentes (Pauline alias Mère Agnès; Marie autre sœur de Thérèse; Mère Marie de Gonzague, prieure du Carmel en 1897) et 
sous trois formes irréductibles. Si le Manuscrit A peut conserver pour C. Langlois le qualificatif d'autobiographique, le manuscrit B devient Le poème de septembre et le manuscrit $\mathrm{C}$ les Lettres à ma Mère bien-aimée.

C. Langlois livre trois commentaires mais aussi, et peut-être surtout, trois éditions, bien que seul le dernier ouvrage paru : L'Autobiographie de Thérèse de Lisieux, assume pleinement le terme d' « édition». Nous y reviendrons, mais il est clair que les deux autres méritent également ce nom, puisque le texte des manuscrits $\mathrm{B}$ et $\mathrm{C} \mathrm{y}$ est bien repris, et sous une forme inédite. Le manuscrit $\mathrm{B}$, rebaptisé Poème de septembre est le texte le plus court, mais commencer par lui une étude qui veut se confronter aux textes de Thérèse jusque dans le détail de leur matérialité relevait de la gageure : outre le flou qui entoure les circonstances et la date exacte de sa composition ${ }^{5}$, un simple regard porté sur les photographies du manuscrit orginal ${ }^{6}$ suffit à faire comprendre l'ampleur de toute tentative d'édition de ce texte. C'est, en effet, à un véritable travail de déchiffrement auquel il faut se livrer non seulement pour comprendre mais déjà pour lire ce " poème " : que faire des innombrables points de suspension utilisés par Thérèse, des majuscules, italiques, grossissements, soulignements, double-soulignements? Claude Langlois prend le pari de tous les justifier, mettant à mal le mythe d'une moniale écrivant au fil de la plume et sans la conscience de l'écrivain. À chaque fois, l'enquête serrée sur les différentes strates rédactionnelles, l'usage des majuscules ou des soulignements, est l'occasion d'une nouvelle prise de vue sur la personnalité ou la spiritualité thérésiennes. Et au final, on retient une trouvaille majeure ${ }^{7}$, qui conduit Claude Langlois à présenter le manuscrit B d'une manière non seulement inédite, mais rendant possible une véritable révolution dans la manière de le lire. Tel, en effet, que présenté dans les autres éditions, le texte est fréquemment haché par des points de suspension, dont on comprend mal la nécessité et qui peuvent témoigner d'une piètre maîtrise thérésienne de la ponctuation. Claude Langlois montre - notamment par des comparaisons avec le style de la sœur dans d'autres écrits - qu'il n'en est

5. Voir Le Poème de septembre, éd. cit., chapitre III, p. 75-101.

6. Reproduites ibid., p. 55-56.

7. Ibid., chapitre II, p. 41-74. 
rien : ces points de suspension sont le moyen typographique trouvé par la moniale pour faire respirer son texte. Ils jouent donc, pour l'auteur, le rôle que les retours à la ligne jouent dans la poésie. De là une présentation originale du texte en poème composé de versets, et qui confère une puissance inattendue à ces « désirs plus grands que l'univers » qu'il exprime, meilleure preuve de la justesse de l'intuition de C. Langlois.

L'ouvrage consacré au "manuscrit $\mathrm{C}$ » s'appuie sur la même démarche : renouveler la lecture du texte à partir d'une étude détaillée du contexte de son écriture et de ses spécificités formelles. Là encore, C. Langlois fait une trouvaille, qui lui permet de le présenter dans une forme plus adéquate que celle du « manuscrit autobiographique ». L'examen attentif, en effet, qui met en évidence des répétitions structurelles cycliques, fait comprendre que Thérèse a écrit chaque jour, et pendant vingt-sept jours de mai-juin 1897, une lettre à sa « Mère bien-aimée », Mère Marie de Gonzague, alors prieure du Carmel de Lisieux; et que donc elle n'a pas pensé son manuscrit comme le récit continu de sa vie au Carmel, bien qu'elle n'ait jamais cessé d'avoir à l'esprit un projet d'ensemble, qu'elle modifiait à mesure qu'elle avançait dans l'écriture. De là le titre du livre de C. Langlois : Lettres à ma Mère bien-aimée, qui présente ce texte comme une correspondance. La découverte, là non plus, n'est pas uniquement formelle. Si elle permet d'expliquer le caractère plutôt décousu de la composition, elle fait en même temps mieux comprendre le rôle du manuscrit pour Thérèse : moyen de supporter la maladie et les doutes, occasion aussi - sans doute attendue et préparée, notamment par des relectures au printemps de ses productions littéraires antérieures - de quotidiens retours sur soi, sur sa vie depuis son entrée au Carmel mais aussi sur la vie du Carmel, avec ce qu'elle porte de douleurs et de tensions. Le commentaire est fouillé, et tous les thèmes abordés. En particulier, les pages difficiles - parmi les plus difficiles du corpus thérésien - sur les tentations contre la foi sont envisagées dans toute leur complexité, et avec une grande originalité. Claude Langlois évite soigneusement l'utilisation anachronique de Thérèse dans le sens d'une théologie de la modernité athée ${ }^{8}$, autant que le lissage de son propos, qui en

8. Voir Jean-François Six, Thérèse de Lisieux par elle-même. 3. L'épreuve et la grâce, Paris, Grasset - Desclée de Brouwer, 1997. 
éliminerait ce qu'il s'y lit de troublant et de provocant pour un esprit croyant. En se concentrant sur la modalité propre à l'écriture de Thérèse dans ces pages, prière plutôt que traité de théologie ou description psychologique, il insiste finalement sur sa « capacité de dire aussi je ne crois pas en évitant le risque du blasphème ${ }^{9} \gg$ qui permet à Thérèse d' '« établir le dialogue dans la nuit même avec l'Être aimé ${ }^{10} »$, mais aussi de reconfigurer profondément sa spiritualité et en particulier son rapport aux pécheurs, avec lesquels elle peut désormais entrer en fraternité.

C. Langlois, enfin, devait se confronter au plus imposant des manuscrits par sa célébrité, sa longueur et son temps de rédaction (de neuf à douze mois). Parue en dernier, l'étude sur le manuscrit $\mathrm{A}$ est à n'en pas douter celle qui fut la plus longue et la plus difficile à mener. Cela, toutefois, est paradoxal, puisqu'on n'y trouve pas, comme dans les deux autres panneaux du triptyque, de proposition formelle vraiment innovante pour la présentation du texte. C'est que le manuscrit de 1895, contrairement aux deux autres, est bien un manuscrit « autobiographique ». Le travail éditorial de Claude Langlois n'est pourtant pas mineur. D'abord, reprenant sa découverte centrale pour le Manuscrit B, et qui était d'ailleurs appuyée sur l'analyse de certains passages du Manuscrit A, il transforme, ici aussi, les points de suspension en renvois à la ligne. Toutefois, parce que leur nombre est bien moindre, et bien qu'ils se multiplient lors de passages clefs ${ }^{11}$, l'innovation n'a pas du tout le même poids. Ensuite, il présente le texte en "séquences » rédactionnelles, de manière à mieux faire apparaître les procédés employés par Thérèse dans son écriture, mais aussi les bouleversements qui l'ont affectée après la rédaction de la parabole des Deux Amours. Enfin, il tâche de restituer le «premier jet » de la rédaction du manuscrit, c'està-dire la version de 1895, avant ajouts de Thérèse ou de son entourage. L'ambition est d'ailleurs toujours la même : « une meilleure approche de l'écriture de Thérèse ${ }^{12} »$, et c'est visiblement cette ambi-

9. Lettres à ma mère bien-aimée..., éd. cit., p. 261.

10. Ibid., p. 257.

11. En particulier lors de la parabole des «Deux amours » (L'Autobiographie..., p. 357-358) dont C. Langlois montre que c'est sa rédaction qui a bouleversé l'ensemble du projet de Thérèse. Nous y reviendrons à propos de l'analyse du dernier livre de la série, consacré à la rivalité amoureuse entre Thérèse et MarieMadeleine.

12. L'Autobiographie..., éd. cit., p. 216. 
tion qui préside aussi à l'adjonction de très nombreuses notes, qui peuvent soit être biographiques, et aider à situer un événement dans la vie de Thérèse ou donner des informations sur des circonstances particulières lors de la rédaction, soit renseigner sur les écarts entre les différentes versions du manuscrit.

Par cette attention explicite à l'écriture de Thérèse, l'édition critique du manuscrit A constitue bien l'aboutissement de toute l'entreprise de C. Langlois. Il faut comprendre qu'en ratifiant l'appellation classique du texte, Claude Langlois ne réduit aucunement ses ambitions d'éditeur. Au contraire, assumer le qualificatif « autobiographique » et le prendre au sérieux lui permet de montrer une Thérèse écrivain ${ }^{13}$ de sa propre vie. Et c'est à cette démonstration que sont consacrés les longs et substantiels chapitres qui précèdent l'édition elle-même. Écrivain, Thérèse l'est parce que loin de se lancer dans l'écriture de ses souvenirs au fil de la remémoration, elle se livre à un vrai travail rédactionnel. Elle réunit des documents - correspondance personnelle, mais aussi correspondances de ses sœurs -, sélectionne les événements en fonction d'un projet initial que C. Langlois va jusqu'à caractériser d'hagiographique, les arrange enfin pour en garder l'essentiel ou mieux les intégrer dans ce projet. Celui-ci bouleversé par la révélation, en juin 1895, de l'amour miséricordieux ${ }^{14}$, elle le reconfigure et n'hésite pas à remanier son manuscrit, ajouter une préface, etc. Mais en montrant un écrivain au travail, rédigeant son autobiographie en forme d'hagiographie implicite, C. Langlois ne vise pas seulement à mettre en évidence la part de fictionnalisation inhérente à toute entreprise de ce genre, bien qu'il note aussi le manque de travaux véritablement biographiques sur la sainte. Il veut plutôt souligner à quel point l'écriture fait partie intégrante de l'expérience spirituelle de Thérèse, que l'écriture de son rapport à Dieu est consubstantielle à ce rapport lui-même, et le désir de sainteté indissociable du désir d'écrire. Dès lors si l'écriture, dans ce qu'elle implique de travail de composition et de rédaction, est l'un des lieux privilégiés de l'expérience spirituelle de Thérèse de Lisieux, quelle autre forme que celle d'une édition critique le meilleur commentaire pourrait-il prendre? Éditer Thérèse

13. Titre d'une conférence donnée par C. Langlois au Centre Sèvres le 8 octobre 2009.

14. L'Autobiographie..., éd. cit., p. 98 et sq. 
n'est donc pas renoncer à proposer une lecture, une interprétation de son texte, mais bien au contraire proposer de son expérience une interprétation comme expérience - entre autres - d'écriture. Il n'y a donc pas contradiction à sous-titrer " édition critique » le volume consacré au Manuscrit A mais « lecture » celui consacré au Manuscrit B comme celui consacré au Manuscrit C. Il s'agit dans les trois cas, et quelle que soit l'importance du travail de traduction du manuscrit en imprimé, d'une même démarche philologique, qui libère le sens du texte en l'affranchissant des formes prises dans sa réception.

Pourquoi, dans ce cas, C. Langlois a-t-il fait paraître, en plus de ces éditions, une autre étude, qui concerne cette fois non plus l'un des manuscrits en particulier mais plutôt un thème récurrent dans toute l'œuvre, retrouvant par certains côtés plutôt la manière qui était la sienne dans Le Désir de Sacerdoce? Ce n'est pas seulement que ce problème de la Rivalité amoureuse entre Thérèse et Marie-Madeleine avait souvent été évoqué dans les ouvrages précédents, à chaque fois rencontré comme un nœud de la spiritualité thérésienne, et que C. Langlois a pu sentir le besoin de s'y confronter directement, de mettre une bonne fois en lumière ce que Marie-Madeleine pouvait représenter pour une carmélite de la fin du XIX ${ }^{e}$ siècle et pour Thérèse de Lisieux en particulier. C'est aussi l'un des meilleurs exemples et comme une ultime confirmation de l'importance de l'écriture pour Thérèse. La figure de MarieMadeleine - pour Thérèse et la plupart des catholiques de l'époque, condensation de Marie de Magdala, Marie de Béthanie et la pécheresse préférée par Jésus au pharisien Simon - est troublante pour la carmélite de Lisieux : elle qui s'estime sans péché trouve devant elle une figure de pécheresse aimée de Jésus, d'autant plus aimée qu'elle a plus péché! Or C. Langlois montre comment c'est l'écriture de la parabole des deux amours qui permet à Thérèse de se libérer, et de libérer son écriture, de l'impasse. En donnant corps à son affrontement avec Marie-Madeleine - et, derrière elle, à sa propre sexualité, le thème du désir thérésien et de son domptage par l'écriture est toujours présent -, cette parabole, théologiquement très problématique, qui met en concurrence l'amour de Dieu qui pardonne les péchés et l'amour de Dieu qui prévient les péchés, permet à Thérèse de dépasser cet affrontement, et du coup, paradoxalement, de s'affranchir de l'obsession de la pureté et de l'écart 
avec les pécheurs, jusqu'à cette ultime fraternité avec eux dans le manuscrit $\mathrm{C}$. La rédaction de la parabole est ainsi découverte pour Thérèse de ce que le travail d'écriture peut lui permettre d'échapper aux difficultés tant théologiques (la question de l'impeccabilité) que psychologiques (le rapport à la sexualité) qui se posent à elle. C'est, peut-être, le moment où Thérèse se découvre écrivain, en ce sens qu'elle découvre comment l'écriture participe de sa vocation.

On l'aura compris, l'apport de C. Langlois aux études thérésiennes - et même plus généralement aux études sur la mystique est inestimable. Ses ouvrages sont des outils de travail désormais incontournables par les méthodes qu'ils mettent en œuvre, la masse d'informations qu'ils contiennent et le visage de la sainte qu'ils dévoilent. Une sainte pleine de désirs contradictoires et brûlants, et qui dépasse ces difficultés dans une écriture imagée pour laquelle elle se passionne. Surtout, ils portent de nouvelles questions : une fois démontrée l'importance de l'acte d'écrire pour Thérèse, ne pourrait-on aller jusqu'à chercher chez elle une théorie de l'écriture spirituelle? Nous serions pour notre part tenté d'en voir une dans la compréhension thérésienne de l'amour comme charme, et dans la valorisation qui s'ensuit d'une capacité à «chanter les Miséricordes du Seigneur ${ }^{15}$ " plutôt que dans celle de l'héroïsme de l'action. Probablement la sainteté est-elle, pour Thérèse, indissociable du charme des saints, comme en témoigne sa fascination pour les saints innocents, dont le seul mérite, précisément, est de posséder le charme de l'enfance ${ }^{16}$. Son chemin de sainteté signifierait, finalement, charme :

« Jésus, à quoi te serviront mes pleurs et mes chants? Ah! je le sais bien, cette pluie embaumée, ces pétales fragiles et sans aucune valeur ces chants d'amour du plus petit des cœurs te charmeront $[\ldots]^{17}$. »

Charmer Dieu et, par là et pour cela, dans un mouvement circulaire puisque la conversion des hommes est pour Thérèse impensable sans la grâce de Dieu, amener les hommes à Dieu en les charmant par le travail d'invention littéraire. En sorte que le travail hagiographique, l'écriture charmante d'une vie, peut lui-même,

15. Ms $\mathrm{C}, \mathrm{F}^{\circ} 1 \mathrm{r}$, dans Lettres à ma Mère bien-aimée..., éd. cit., p. 137.

16. Voir notamment la poésie «À mes petits frères du Ciel » (PN 44, dans Euvres Complètes de Thérèse de Lisieux, Paris, Cerf, 2006).

17. Ms B, F० $4 \mathrm{v}$, dans Le Poème de septembre, éd. cit., p. 65 . 
pour Thérèse, être saint, dans la mesure où il participe de son travail de conversion par lequel elle prétend charmer Dieu. Une étude systématique serait à mener de ce point, et en particulier à partir de tout ce pan des écrits de Thérèse laissés largement inexplorés par Claude Langlois : les « récréations pieuses » et les poésies écrites pour le Carmel.

La découverte de l'importance des textes, dans leur matérialité même, pour la compréhension de la spiritualité spécifiquement thérésienne, pointe également vers une autre direction, d'ailleurs liée à cette dimension du charme : le problème de la réception et de la diffusion des textes de Thérèse. Dans quelle mesure la sainte a-telle eu conscience, voire orchestré elle-même sa postérité? Si elle fut écrivain, jusqu'où a-t-elle pensé être lue? Quelle ont été les motivations des sœurs du Carmel de Lisieux pour diffuser à ce point ses textes et jusqu'où ont été, pour cela et pour quels autres motifs, les adaptations, voire les mystifications? Autant de questions dont la nécessité naît aussi du travail de C. Langlois, qui met le texte au cœur de l'expérience thérésienne. Dans ces conditions, comprendre jusqu'au bout cette expérience - qui dépasse, on le comprend, la seule personnalité de Thérèse, puisque comme le montre C. Langlois les écrits de la moniale sont, dès le début, pris dans des réseaux d'inconscients, et jouent toujours un rôle non seulement pour Thérèse, mais aussi pour ceux à qui ils s'adressent, ou répondent - ce sera, nécessairement, suivre les aventures de ces textes dans le $\mathrm{xx}^{\mathrm{e}}$ siècle, depuis le lit de mort où l'on consignait ses derniers propos, dans les environs de Lisieux, les paroisses catholiques du début des années 1900, dans les tranchées de la Grande Guerre et au moins jusqu'à la canonisation de 1927, peut-être au-delà. Vaste travail, aussi nécessaire pour l'historien du thérésianisme que pour le philosophe de l'expérience mystique thérésienne.

anthony.feneuil@unige.ch 